\title{
Adaptive Fuzzy Observer based Hierarchical Sliding Mode Control for Uncertain 2D Overhead Cranes
}

\author{
Hai Xuan Le $e^{\mathrm{a}}$, Anh Viet Le ${ }^{\mathrm{a}}$ and Linh Nguyen ${ }^{\mathrm{b}}$ ** \\ ${ }^{a}$ Department of Automatic Control, Hanoi University of Science and Technology, 1 Dai \\ Co Viet, Hai Ba Trung, Hanoi, Vietnam, 10000 \\ ${ }^{b}$ Centre for Autonomous Systems, University of Technology Sydney, 15 Broadway, \\ Ultimo, NSW, Australia, 2007
}

"Corresponding Author / E-mail: vanlinh.nguyen@uts.edu.au, TEL: +61-2-9514-1225, FAX: +61-2-9514-2655, ORCID iD: 0000-0001-5360-886X

Hai Xuan Le received his $\mathrm{PhD}$ degree in Automatic Control from Hanoi University of Science and Technology, Vietnam in 2019, where he is currently a researcher at the Department of Automatic Control. His research interests include non-linear adaptive control for under-actuated systems, fuzzy logic inference and neural network, programmable logic controller in industry.

Anh Viet Le is currently a research assistant at the Department of Automatic Control, Hanoi University of Science and Technology, Hanoi, Vietnam. His research interests include non-linear adaptive control for under-actuated systems, fuzzy logic inference and neural network, microcontroller and applications.

Linh Nguyen received his $\mathrm{PhD}$ degree in Robotics from the University of Technology Sydney (UTS), Australia in March 2015. He then worked at the same university as a Postdoctoral Research Associate until September 2015. From January to July 2016, he was as Research Fellow at the School of Electrical and Electronic Engineering, Nanyang Technological University, Singapore. He rejoined the Centre for Autonomous Systems at UTS in August 2016, where he is currently a Research Fellow. His research interest includes robotics, internet of robotic things, artificial intelligence, machine learning, signal processing, control theory and non-destructive testing. 


\title{
Adaptive Fuzzy Observer based Hierarchical Sliding Mode Control for Uncertain 2D Overhead Cranes
}

\begin{abstract}
This paper proposes a new approach to robustly control a 2D under-actuated overhead crane system, where a payload is effectively transported to a destination in real time with small sway angles, given its inherent uncertainties such as actuator nonlinearities and external disturbances. The control law is proposed to be developed by the use of the robust hierarchical sliding mode control (HSMC) structure in which a second-level sliding surface is formulated by two first-level sliding surfaces drawn on both actuated and under-actuated outputs of the crane. The unknown and uncertain parameters of the proposed control scheme are then adaptively estimated by the fuzzy observer, where the adaptation mechanism is derived from the Lyapunov theory. More importantly, stability of the proposed strategy is theoretically proved. Effectiveness of the proposed adaptive fuzzy observer based HSMC (AFHSMC) approach was extensively validated by implementing the algorithm in both synthetic simulations and real-life experiments, where the results obtained by our method are highly promising.
\end{abstract}

Keywords: 2D overhead crane, hierarchical sliding mode control, fuzzy observer, under-actuated systems, Lyapunov function

\section{Introduction}

An overhead crane, also called a bridge crane, is more and more popular in many manufactures or industries due to its universal ability to transport a heavy object or hazardous material from one place to another place. It is also widely utilized in harbour ports to load and unload cargo from ships [1,2]. The model of the overhead crane is classified into a class of under-actuated mechanical systems, where a number of control inputs is less than a number of actuators. For instance, in a 2D overhead crane, both the position of the trolley and the swing angle of an object is dependent on the control force. Due to oscillations of the payload during operations, the bridge crane may severely cause 
unsafety on operators, loads or surrounding items [3]. Furthermore, the oscillation of the payload is nonlinear and highly coupled with motion of the trolley, which make the overhead crane cumbersome to control. As a result, expectations in controlling the 2D overhead crane are to effectively drive the trolley on a desired path while minimizing the swing angle of the payload.

Many approaches to efficiently control an overhead crane have been proposed in the past decades, from the open-loop to closed-loop control techniques. While the openloop control method [4] is straightforward to implement on a crane but very sensitive to external disturbances, the closed-loop approaches, by employing sensor measurements and system state estimations, are more efficient to control crane actuators due to their less sensitivity to the external disturbances as well as system uncertainties [5]. For instance, Yu et al. [6] proposed a nonlinear two closed loop controller to fast transport an object to a desired destination while minimizing its oscillations. Another nonlinear control law based on partial feedback linearization was developed for a 2D crane system [7]. Furthermore, in the works $[8,9]$, Le et al. extended the nonlinear partial feedback linearization controllers for a 3D overhead crane system by considering nonlinear feedback of actuated and un-actuated states in a superposition fashion. Due to strong adaptability to complexity and nonlinearity of a crane, a fuzzy model was used to present an overhead crane, which leads to a fuzzy logic controller in the works [10,11].

To maintain robustness of a crane system under its actuator nonlinearities and parameter uncertainties, sliding mode control (SMC) has attracted much attention from researchers and practitioners [12-14]. However, how to formulate the sling surfaces so as to guarantee stability of actuators and un-actuators in a crane is really challenging. For instance, the work [15] first developed an intermediate variable based upon state errors and then formed as second-level sliding surface. Among approaches of defining a sliding 
surface for a SMC scheme, the hierarchical SMC (HSMC) law has been widely employed in a class of under-actuated systems [16,17,27,28,33]. Wang et al. [16] proposed the HSMC strategy for a class of second-order under-actuated systems, where a first-level sliding surface is defined for each subsystem. A second-level sliding surface is then formulated as a linear combination of the first-level sliding surfaces. Similarly, the authors of the work [17] developed another hierarchical structure of sliding surfaces in designing a control law for a single-input-multiple-output under-actuated system. The first-layer sliding surface based on the first subsystem is computed, which is then incorporated into the sliding surface of a second subsystem to formulate a second-layer sliding surface. The chain is subsequently produced until the last subsystem.

It is noted that in the SMC laws all the system parameters are assumed to be known and certain. Though, in fact, those parameters can be deterministically estimated, they are highly uncertain due to the system nonlinearities and external disturbances. That is, it is impractical to certainly determine the system parameters Therefore, the more accurately the system parameters are computed, the more efficiently the control scheme works. Some adaptive control strategies have been developed to consider the system parameter uncertainties. For instance, in the works [18,19], a nonlinear coupling control law for a crane system is discussed, where its parameters are adaptively updated. Likewise, Le et al. [20] proposed a model reference adaptation mechanism, derived from Lyapunov theory, to approximate system parameters in a crane system. Moreover, a fuzzy disturbance observer was introduced by Kim [21] for designing a controller in nonlinear systems. By employing the fuzzy observer [21], Park et al. [22,23] discussed a method to represent crane system uncertainties as well as actuator nonlinearities. In the previous work [30], we considered a SMC law for an offshore container crane, where system parameters are adaptively learned by a radial basis function network. Moreover, in the 
work [32], Wang et al. employed a finite-time disturbance observer to identify unknown disturbances in complex marine environments. The proposed observer is utilized in conjunction with the nonsingular fast terminal sliding mode manifold to form a new finite-time control law for effectively tracking trajectories of a surface vehicle. Some other SMC strategies were designed for non-linear systems in which unknown parameters are adaptively estimated by a neural network [24,29], a fuzzy-neural network [25] and a fuzzy wavelet neural network [26].

In this work, an adaptive HSMC strategy employing the fuzzy observer is investigated to address the control problem in a 2D overhead crane system. There are two hierarchical layers in the proposed SMC law. Two first-level sliding surfaces are formulated from two subsystems, including actuated and under-actuated outputs of the 2D overhead crane. Then the sliding surface in the second layer is simply formed by linearly combining two first-level sliding surfaces. Moreover, to deal with challenges in computing the system parameters due to system uncertainties such as parameter variations and nonlinearities or external disturbances, it is proposed to utilize the fuzzy observer to approximately but adaptively estimate the dynamics of the crane. The adaptive fuzzy observer based HSMC (AFHSMC) algorithm was extensively validated in both synthetic simulations and real-life experiments. The results obtained by the proposed approach are highly promising. More importantly, based on the Lyapunov theory, stability of the $2 \mathrm{D}$ overhead crane system is theoretically proven.

The remaining of the paper is arranged as follows. The dynamic model of a $2 \mathrm{D}$ overhead crane is described in Section 2 while the conventional HSMC scheme is introduce in Section 3. Section 4 discusses the proposed AFHSMC law, where stability of the system is theoretically analyzed. Validations of the proposed method in both the simulations and experiments are delineated in Section 5 before conclusions are drawn in 
Section 6.

\section{Dynamic Model of 2D Overhead Crane}

Let's consider an overhead crane on a 2D $x y$ plane as demonstrated in Fig. 1. The system comprises a trolley moving on a rail, a payload that is considered as a point mass and a hoisting cable. It is assumed that the static friction and elasticity are trivial while stiffness and mass of the hoisting cable are neglected. Moreover, both the trolley and payload are considered as material particles.

We denote $x, \theta$ and $F$ are variables presenting for the trolley position, the swing angle of the payload with respect to the vertical line and the trolley driving force, respectively. On the other hands, $M, m$ and $l$ are also defined as constant parameters delineating the total mass of the trolley, the mass of the payload and the hoisting cable length. Furthermore, in this work, it is assume that the overhead crane operates in indoor environments, hence the effects of disturbances caused by wind are not examined. Then, the mathematical dynamic model of the 2D overhead crane [5] can be specified by

$$
\begin{gathered}
(M+m) \ddot{x}+m l \ddot{\theta} \cos \theta-m l \dot{\theta}^{2} \sin \theta=F, \\
l \ddot{\theta}+g \sin \theta+\ddot{x} \cos \theta=0,
\end{gathered}
$$

where $g$ is the gravitational acceleration. It is noted that in the state space, if we let $u=F$

and $X^{T}=\left[\begin{array}{llll}x_{1} & x_{2} & x_{3} & x_{4}\end{array}\right]=\left[\begin{array}{llll}x & \dot{x} & \theta & \dot{\theta}\end{array}\right]$, then the dynamic model can be represented as follows,

where

$$
\begin{gathered}
\dot{x}_{1}=x_{2}, \\
\dot{x}_{2}=f_{1}(X)+g_{1}(X) u, \\
\dot{x}_{3}=x_{4}, \\
\dot{x}_{4}=f_{2}(X)+g_{2}(X) u,
\end{gathered}
$$

$$
f_{1}(X)=\frac{m l \dot{\theta}^{2} \sin \theta+m g \sin \theta \cos \theta}{M+m \sin ^{2} \theta},
$$




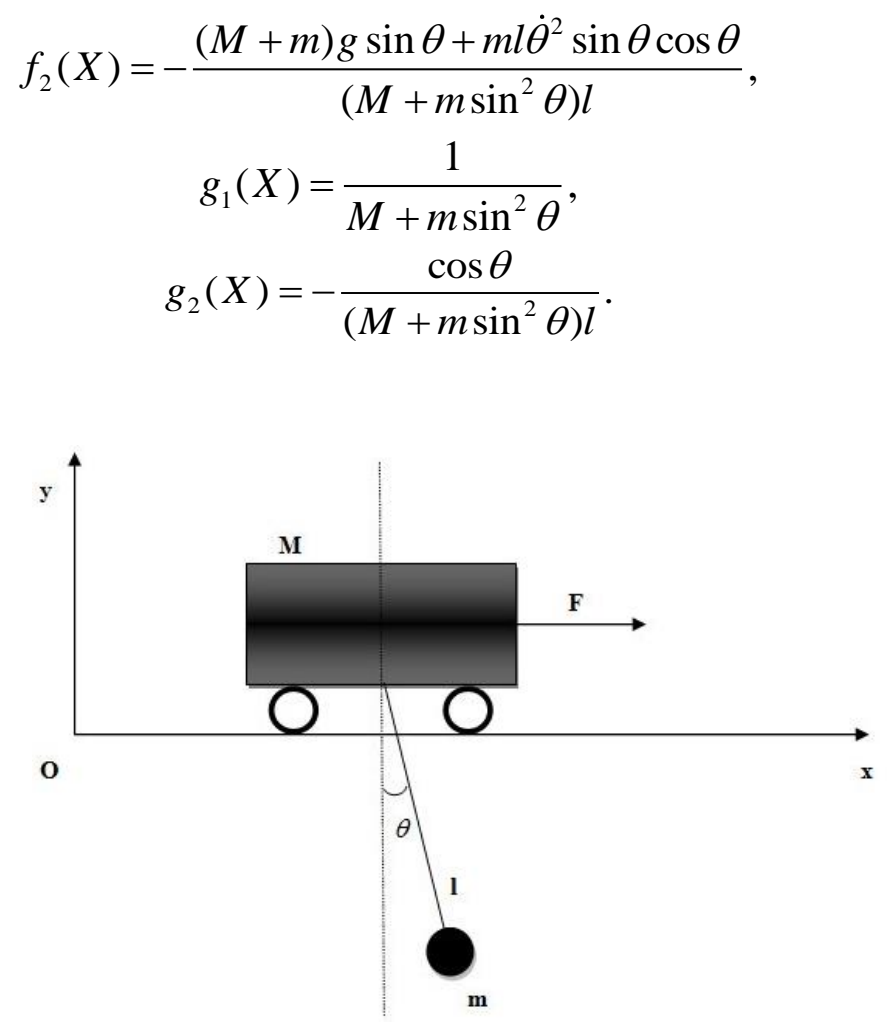

Figure 1. Dynamic model of a 2D overhead crane

It can be clearly seen that there is one input as the driving force while there are two outputs as the motion of the trolley and the swing angle of the payload. Therefore, the primary difficulty in controlling an under-actuated overhead crane is how to manage the coupled nature of the outputs from a single input.

\section{Hierarchical Sliding Mode Control}

In order to overcome issues in controlling an overhead crane system, many control strategies have been proposed, including feedback linearization [6,8], fuzzy control $[10,11]$, and adaptive control [18,21,22]. Nonetheless, the control designers have remarkably paid more attention on the SMC approach due to its well-known robustness under uncertain conditions. Among the SMC schemes, a hierarchical SMC (HSMC) has been considered as an appropriate approach for controlling a class of under-actuated systems [17]. In this sections, we discuss the HSMC strategy for a 2D overhead crane, which will be then utilized to adapt to its adaptive paradigm in the next section. 
To ascertain the stability of the sliding surfaces, based on the Lyapunov theory, let's define the vectors of the errors as follows,

$$
e(t)=\left[\begin{array}{l}
x_{1}-x_{d} \\
x_{3}-\theta_{d}
\end{array}\right]=\left[\begin{array}{l}
x-x_{d} \\
\theta-\theta_{d}
\end{array}\right]=\left[\begin{array}{l}
e_{1} \\
e_{3}
\end{array}\right]
$$

where $x_{d}$ and $\theta_{d}$ are the desired trolley position and swing angle of the payload. It is assumed that $\dot{x}_{d}$ and $\ddot{x}_{d}$ exist and are bounded, then derivatives of the errors derived from (2) can be specified by,

$$
\begin{gathered}
\dot{e}_{1}=e_{2}, \\
\dot{e}_{2}=f_{1}(X)+g_{1}(X) u-\ddot{x}_{d}, \\
\dot{e}_{3}=e_{4}, \\
\dot{e}_{4}=f_{2}(X)+g_{2}(X) u,
\end{gathered}
$$

Now, we define two first-level sliding surfaces for the corresponding actuated output $x$ and under-actuated output $\theta$ as follows,

$$
\begin{aligned}
& s_{1}=c_{1} e_{1}+e_{2}, \\
& s_{2}=c_{2} e_{3}+e_{4},
\end{aligned}
$$

where $c_{1}$ and $c_{2}$ are positive constants. The second-level sliding surface for the whole system is then aggregated by

$$
s=\alpha s_{1}+\beta s_{2},
$$

where $\alpha$ and $\beta$ are positive parameters. Note that if an appropriate HSMC scheme is given, the second-level sliding surface can ultimately converge to zero. As pointed out by Qian et al. [13], such SMC scheme should comprise two laws. The first is the switch control law that is utilized to drive the system states towards a particular sliding surface, while the second is the equivalent control law that is employed to keep those states on the sliding surface. In other words, the HSMC scheme can be presented by

$$
u=u_{e q}+u_{s w} .
$$

To design that SMC law, let's compute the derivative of the second-level sliding surface from (5) and (6) as follows, 


$$
\begin{aligned}
\dot{s} & =\alpha \dot{s}_{1}+\beta \dot{s}_{2} \\
& =\alpha\left(c_{1} e_{2}+f_{1}+g_{1} u-\ddot{x}_{d}\right)+\beta\left(c_{2} e_{4}+f_{2}+g_{2} u\right) .
\end{aligned}
$$

If we consider the Lyapunov function candidate as

$$
V=\frac{1}{2} s^{2}
$$

and differentiate it with respect to time, we obtain

$$
\begin{aligned}
\dot{V} & =s \dot{s} \\
& =s\left(\alpha\left(c_{1} e_{2}+f_{1}+g_{1} u-\ddot{x}_{d}\right)+\beta\left(c_{2} e_{4}+f_{2}+g_{2} u\right)\right) \\
& =s\left(\alpha c_{1} e_{2}+\beta c_{2} e_{4}+\alpha f_{1}+\beta f_{2}-\alpha \ddot{x}_{d}+\left(\alpha g_{1}+\beta g_{2}\right) u\right) .
\end{aligned}
$$

In order to guarantee the stability of the second-level sliding surface, we define

$$
\left\{\begin{array}{l}
\left(\alpha g_{1}+\beta g_{2}\right) u_{e q}+\alpha c_{1} e_{2}+\beta c_{2} e_{4}+\alpha f_{1}+\beta f_{2}-\alpha \ddot{x}_{d}=0, \\
\left(\alpha g_{1}+\beta g_{2}\right) u_{s \mathrm{w}}+k_{1} s+k_{2} \operatorname{sgn}(s)=0,
\end{array}\right.
$$

where $k_{1}$ and $k_{2}$ are positive. Therefore, the HSMC scheme is given by

$$
u=-\frac{\alpha c_{1} e_{2}+\alpha f_{1}+\beta c_{2} e_{4}+\beta f_{2}-\alpha \ddot{x}_{1 d}+k_{1} s+k_{2} \operatorname{sgn}(s)}{\alpha \hat{g}_{1}+\beta \hat{g}_{2}},
$$

where $f_{1}, g_{1}, f_{2}$ and $g_{2}$ rely on the system parameters, comprising $M, m, l, \theta$, as presented in (3). If substituting (10) into (9), we obtain

$$
\dot{V}=s \dot{s}=s\left(-k_{1} s-k_{2} \operatorname{sgn}(\mathrm{s})\right)=-k_{1} s^{2}-k_{2}|s| \leq 0,
$$

which guarantees that the sliding mode is reachable in finite time.

\section{Fuzzy observer based adaptive hierarchical sliding mode control}

It can be clearly seen that the deterministic HSMC scheme introduced in Section 3 can be effectively employed provided that the system parameters are certain. Nevertheless, in practice, an overhead crane operates under system uncertainties such as parameter variations and nonlinearities or external disturbances; that is, the dynamic models presented in the previous sections do not fully delineate the characteristics of the crane system. In other words, it is impractical to accurately determine the system parameters. To deal with these challenges, it is proposed to utilize the fuzzy observer to approximately estimate those dynamic models. In this section, we first introduce the fuzzy observer, 
which is then incorporated into the HSMC law to design an adaptive controller for a $2 \mathrm{D}$ overhead crane.

\subsection{Fuzzy observer}

Let's consider approximation of a fuzzy system as introduced by Park et al. [22]. A fuzzy system comprises three fundamental elements including fuzzifier, fuzzy inference engine and defuzzifier. The fuzzy inference engine employs the fuzzy IF-THEN rules to compute fuzzy sets in the input space to fuzzy sets in the output space. Moreover, while the fuzzifier is considered to map a real-valued point to fuzzy set, the defuzzifier maps a fuzzy set as an output of the fuzzy inference engine to a crisp point. For instance, in this work, we propose to employ the fuzzy IF-THEN rules, whose $i^{\text {th }}$ law is specified by

$$
\text { If } x_{1} \text { is } A_{1}^{i}, x_{2} \text { is } A_{2}^{i}, \ldots, x_{n} \text { is } A_{n}^{i} \text {, then } y \text { is } y^{i} \text {, }
$$

where $x=\left(x_{1}, x_{2}, \ldots, x_{n}\right) \in R^{n}$ is the input of the fuzzy system. $A_{j}^{i}$ the $i^{\text {th }}$ fuzzy set of the input variable $x_{j}, y \in R$ is the output of the fuzzy system, where its element $y^{i}$ is a singleton number, $i=1,2, \ldots, r$ and $j=1,2, \ldots, n$.

If the fuzzy system utilizes product inference engine, singleton fuzzifier and center average defuzzifier, its output can be presented in the following form.

$$
y(x)=\frac{\sum_{i=1}^{r} y^{i}\left(\prod_{j=1}^{n} \mu_{A_{j}^{i}}\left(x_{j}\right)\right)}{\sum_{i=1}^{r}\left(\prod_{j=1}^{n} \mu_{A_{j}^{i}}\left(x_{j}\right)\right)}=\hat{\phi}^{T} \xi(x),
$$

where, $\mu_{A_{j}^{i}}\left(x_{j}\right)$ is the membership function of the fuzzy set $A_{j}^{i} . \xi^{T}=\left(\xi^{1}, \xi^{2}, \ldots, \xi^{r}\right)$, whose component is computed by

$$
\xi^{i}=\frac{\prod_{j=1}^{n} \mu_{A_{j}^{i}}\left(x_{j}\right)}{\sum_{i=1}^{r}\left(\prod_{j=1}^{n} \mu_{A_{j}^{i}}\left(x_{j}\right)\right)},
$$

which is the fuzzy basic function. And $\hat{\phi}^{T}=\left(y^{1}, y^{2}, \ldots, y^{r}\right)$ is a vector of the adjustable parameters. 
In a nonlinear system, if there exist an adjusted $\hat{\phi}^{T}$ so that a nonlinearity $f(x)$ approximates to $y(x)$, then $|f-y|$ is minimal. In equivalent words, in that case, the nonlinearity $f(x)$ can be approximately estimated by an output of a fuzzy system.

In the overhead system, the four dynamic models (3) as presented in the previous section are nonlinear, unknown and uncertain, which is not trivial to practically determine. Thus, we propose to estimate them by the use of the fuzzy systems. In other words, a fuzzy observer (FO) to approximate $f_{1}(X), g_{1}(X), f_{2}(X)$ and $g_{2}(X)$ is defined as follows,

$$
\left\{\begin{array}{l}
f_{1}(X)=\phi_{1}^{T} \xi(\mathrm{X})+\varepsilon_{1}(X) \\
g_{1}(X)=\phi_{2}{ }^{T} \xi(\mathrm{X})+\varepsilon_{2}(X) \\
f_{2}(X)=\phi_{3}{ }^{T} \xi(\mathrm{X})+\varepsilon_{3}(X) \\
g_{2}(X)=\phi_{4}{ }^{T} \xi(\mathrm{X})+\varepsilon_{4}(X)
\end{array}\right.
$$

where $\phi_{i}$ is the ideal adjustable parameter, and $\left|\varepsilon_{i}(\mathrm{X})\right| \leq \varepsilon_{i}$ is the approximation error with constant $\varepsilon_{i}>0, i=1,2,3,4$.

\subsection{FO based adaptive HSMC scheme}

Let $\hat{\phi}_{1}, \hat{\phi}_{2}, \hat{\phi}_{3}$ and $\hat{\phi}_{4}$ denote estimations of $\phi_{1}, \phi_{2}, \phi_{3}$ and $\phi_{4}$, respectively. Then the outputs of the fuzzy observer are approximations of the dynamics $f_{1}, g_{1}, f_{2}$ and $g_{2}$ and computed as follows,

$$
\left\{\begin{array}{l}
\hat{f}_{1}(X)=\hat{\phi}_{1}^{T} \xi(X) \\
\hat{g}_{1}(X)=\hat{\phi}_{2}^{T} \xi(X) \\
\hat{f}_{2}(X)=\hat{\phi}_{3}^{T} \xi(X) \\
\hat{g}_{2}(X)=\hat{\phi}_{4}^{T} \xi(X) .
\end{array}\right.
$$

We now introduce how to employ the fuzzy system approximations $\hat{f}_{1}, \hat{g}_{1}, \hat{f}_{2}$ and $\hat{g}_{2}$ in designing the adaptive HSMC law. 
Let's consider the Lyapunov function candidate

$$
V=\frac{1}{2}\left(s^{2}+\sum_{i=1}^{4} \tilde{\phi}_{i}^{T} \Gamma_{i}^{-1} \tilde{\phi}_{i}\right),
$$

where $\tilde{\phi}_{i}=\phi_{i}-\hat{\phi}_{i}$ is the error between ideal and estimated adjustable parameters. $\Gamma_{i}$ is positive constant.

If we differentiate the Lyapunov function candidate in (15), it yields,

$$
\dot{V}=s \dot{s}+\sum_{i}^{4} \tilde{\phi}_{i}^{T} \Gamma_{i}^{-1} \dot{\tilde{\phi}}
$$

In a more specific form, (16) can be presented as follows,

$$
\begin{aligned}
\dot{V}= & s\left(\alpha\left(c_{1} e_{2}+f_{1}+g_{1} u-\ddot{x}_{1 d}\right)+\beta\left(c_{2} e_{4}+f_{2}+g_{2} u\right)\right) \\
& +\sum_{i}^{4} \tilde{\phi}_{i}^{T} \Gamma_{i}^{-1} \dot{\tilde{\phi}}_{i} \\
= & s\left(\alpha c_{1} e_{2}+\alpha \hat{f}_{1}+\beta c_{2} e_{4}+\beta \hat{f}_{2}-\ddot{x}_{1 d}+\left(\alpha \hat{g}_{1}+\beta \hat{g}_{2}\right) u\right) \\
& +s\left(\alpha\left(\tilde{\phi}_{1}^{T} \xi+\varepsilon_{1}\right)+\beta\left(\tilde{\phi}_{3}^{T} \xi+\varepsilon_{3}\right)+\alpha u\left(\tilde{\phi}_{2}^{T} \xi+\varepsilon_{2}\right)\right) \\
& +s\left(\beta u\left(\tilde{\phi}_{4}^{T} \xi+\varepsilon_{4}\right)\right)+\sum_{i}^{4} \tilde{\phi}_{i}^{T} \Gamma_{i}^{-1} \dot{\tilde{\phi}}_{i}
\end{aligned}
$$

Moreover, given the estimations $\hat{f}_{1}, \hat{g}_{1}, \hat{f}_{2}$ and $\hat{g}_{2}$ obtained by the FO, the control signal of the HSMC scheme in (10) can be approximately computed by

$$
u=-\frac{\alpha c_{1} e_{2}+\alpha \hat{f}_{1}+\beta c_{2} e_{4}+\beta \hat{f}_{2}-\alpha \ddot{x}_{1 d}+k_{1} s+k_{2} \operatorname{sgn}(s)}{\alpha \hat{g}_{1}+\beta \hat{g}_{2}}
$$

From (16) to (18), the derivative of the Lyapunov function candidate can be rewritten as follows,

$$
\begin{aligned}
\dot{V}= & -k_{1} s^{2}-k_{2} \operatorname{sgn}(s) . s+\sum_{i}^{4} \tilde{\phi}_{i}^{T} \Gamma_{i}^{-1} \dot{\tilde{\phi}}_{i} s \\
& +\alpha\left(\tilde{\phi}_{1}^{T} \xi+\varepsilon_{1}\right)+\beta\left(\tilde{\phi}_{3}^{T} \xi+\varepsilon_{3}\right) \\
& +\alpha u\left(\tilde{\phi}_{2}^{T} \xi+\varepsilon_{2}\right)+\beta u\left(\tilde{\phi}_{4}^{T} \xi+\varepsilon_{4}\right) \\
= & -k_{1} s^{2}-k_{2}|s|+\tilde{\phi}_{1}^{T}\left(\alpha s \xi+\Gamma_{1}^{-1} \dot{\tilde{\phi}}\right)+\tilde{\phi}_{2}^{T}\left(\alpha u s \xi+\Gamma_{2}^{-1} \dot{\tilde{\phi}}_{2}\right) \\
& +\tilde{\phi}_{3}^{T}\left(\beta s \xi+\Gamma_{3}^{-1} \dot{\tilde{\phi}}_{3}\right)+\tilde{\phi}_{4}^{T}\left(\beta u s \xi+\Gamma_{4}^{-1} \dot{\tilde{\phi}}_{4}\right) \\
& +s\left(\alpha \varepsilon_{1}+\beta \varepsilon_{3}+\alpha u \varepsilon_{2}+\beta u \varepsilon_{4}\right)
\end{aligned}
$$

If the approximations of the adjustable parameters, including $\hat{\phi}_{1}, \hat{\phi}_{2}, \hat{\phi}_{3}$ and $\hat{\phi}_{4}$, are adaptively selected, where their derivatives are formulated by 


$$
\begin{gathered}
\dot{\hat{\phi}}_{1}=\Gamma_{1} \alpha s \xi, \\
\dot{\hat{\phi}}_{2}=\Gamma_{2} \alpha u s \xi, \\
\dot{\hat{\phi}}_{3}=\Gamma_{3} \beta s \xi, \\
\dot{\hat{\phi}}_{4}=\Gamma_{4} \alpha u s \xi,
\end{gathered}
$$

then (19) can be simplified as follows,

$$
\dot{V}=-k_{1} s^{2}-k_{2}|s|+s\left(\alpha \varepsilon_{1}+\beta \varepsilon_{3}+\alpha u \varepsilon_{2}+\beta u \varepsilon_{4}\right)
$$

We assume that $\left|\alpha \varepsilon_{1}+\beta \varepsilon_{3}+\alpha u \varepsilon_{2}+\beta u \varepsilon_{4}\right| \leq \varepsilon_{N}$, where $\varepsilon_{N}$ is a small positive number, if $k_{2}-\varepsilon_{N}>0$ then $\dot{V} \leq 0$.

It can be clearly seen that given the stability conditions based upon the Lyapunov function, the adaptation mechanism in (20) allows the fuzzy system to adaptively estimate dynamics of the overhead crane as presented in (14), which ultimately leads to the adaptive control law in (18).

\subsection{Stability analysis}

The effectiveness of the proposed FO based adaptive HSMC (AFHSMC) scheme for a 2D overhead crane can be mathematically demonstrated by stability of the closed loop control system in the following theorem.

Theorem 1: Given the adaptation mechanism (20), the FO-AHSMC law (18) can asymptotically stabilize the second-level sliding surface $s$ defined in (5) if $k_{2}-\varepsilon_{N}>0$.

Proof: Let's take integrals of both sides of (21), it yields,

$$
\begin{array}{r}
\int_{0}^{t} d V=\int_{0}^{t}\left(-k_{1} s^{2}-k_{2}|s|+s\left(\alpha \varepsilon_{1}+\beta \varepsilon_{3}+\alpha u \varepsilon_{2}+\beta u \varepsilon_{4}\right)\right) d t, \\
V(\mathrm{t})-\mathrm{V}(0)=\int_{0}^{t}\left(-k_{1} s^{2}-k_{2}|s|+s\left(\alpha \varepsilon_{1}+\beta \varepsilon_{3}+\alpha u \varepsilon_{2}+\beta u \varepsilon_{4}\right)\right) d t,
\end{array}
$$

or

$$
\begin{aligned}
V(0) & =V(t)+\int_{0}^{t}\left(k_{1} s^{2}+k_{2}|s|-s\left(\alpha \varepsilon_{1}+\beta \varepsilon_{3}+\alpha u \varepsilon_{2}+\beta u \varepsilon_{4}\right)\right) d t \\
& \geq \int_{0}^{t}\left(k_{1} s^{2}+\left(k_{2}-\varepsilon_{N}\right)|s|\right) d t
\end{aligned}
$$

In other words, 


$$
\begin{aligned}
V(t) & =\frac{1}{2} s^{2} \\
& \leq V(0)-\int_{0}^{t}\left(k_{1} s^{2}+\left(k_{2}-\varepsilon_{N}\right)|s|\right) d t \leq V(0)<\infty
\end{aligned}
$$

Therefore, we have

$$
s \in L_{\infty} \text {, i.e. } \sup _{t \geq 0}|s|=\|s\|_{\infty}<\infty .
$$

Moreover, from (21) it shows that

$$
\begin{aligned}
|\dot{V}| & =|\dot{s}||s| \\
& \leq k_{1} s^{2}+k_{2}|s|+s\left(\alpha \varepsilon_{1}+\beta \varepsilon_{3}+\alpha u \varepsilon_{2}+\beta u \varepsilon_{4}\right) \\
& \leq k_{1} s^{2}+k_{2}|s|+|s| \varepsilon_{N},
\end{aligned}
$$

which results in

$$
|\dot{s}| \leq k_{1}|s|+k_{2}+\varepsilon_{N}<\infty
$$

Hence,

$$
\dot{s} \in L_{\infty} \text {, i.e. } \sup _{t \geq 0}|\dot{s}|=\|\dot{s}\|_{\infty}<\infty .
$$

(23) can now be rewritten by

$$
\begin{aligned}
V(0) & \geq \int_{0}^{\infty}\left(k_{1} s^{2}+\left(k_{2}-\varepsilon_{N}\right)|s|\right) d t \\
& =\int_{0}^{\infty} k_{1} s^{2} d t+\int_{0}^{\infty}\left(k_{2}-\varepsilon_{N}\right)|s| d t .
\end{aligned}
$$

It is apparent that $\int_{0}^{\infty} k_{1} s^{2} d t \geq 0$ and $\int_{0}^{\infty}\left(k_{2}-\varepsilon_{N}\right)|s| d t \geq 0$. From (28) it shows $\int_{0}^{\infty}|s| d t<\infty$ , i.e. $\|s\|_{1}<\infty$, and $\int_{0}^{\infty} s^{2} d t<\infty$, i.e. $s \in L_{2}$.

Since $s \in L_{\infty}, \dot{s} \in L_{\infty}$, and $s \in L_{2}$, according to Barbalat's lemma [31] we have $\lim _{t \rightarrow \infty} s=0$

. Therefore the second-level sliding surface $s$ is asymptotically stable.

\section{Simulation and experimental results}

In order to demonstrate efficiency of the proposed AFHSMC approach as compared with that of the conventional HSMC, we conducted the experiments both on a synthetic simulation environment and in a laboratory. It is noted that due to impracticality of determining the uncertain parameters of the dynamics of the overhead crane, we did not 
implement the HSMC law in the realistic system. Nevertheless, in the synthetic simulation, the deterministic HSMC scheme was expected to best perform since the system parameters were accurately obtained.

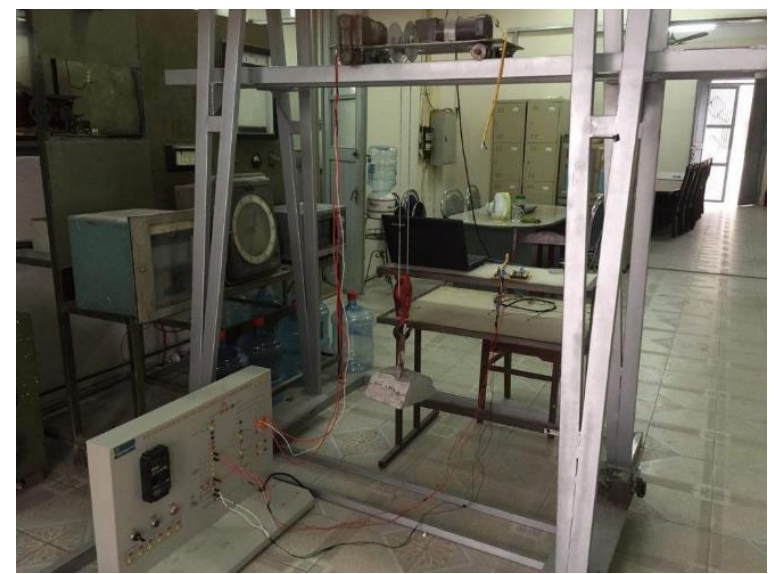

Figure 2. A real-time overhead crane system

In the first experiment, we conducted a simulation of a realistic overhead crane system as pictorially demonstrated in Fig. 2. To this end, the physical parameters of the laboratory overhead crane are summarized as follows,

$$
M=25 \mathrm{~kg}, m=8 \mathrm{~kg}, l=1.2 \mathrm{~m}, g=9.81 \mathrm{~m} / \mathrm{s}^{2}
$$

Furthermore, in the experiments, some positive constant parameters of the control laws were given by

$$
c_{1}=3, c_{1}=0.01, \alpha=2, \beta=1.4, \mathrm{k}_{1}=0.1, k_{2}=2 \text {. }
$$

Table 1. Parameters of the membership functions

\begin{tabular}{c|c|c|c|c}
\hline \multirow{2}{*}{$i$} & \multicolumn{2}{|c|}{$\mu_{A_{1}^{i}}(\theta)$} & \multicolumn{2}{c}{$\mu_{A_{2}^{i}}(\dot{\theta})$} \\
\cline { 2 - 5 } & $\sigma$ & $v[\mathrm{rad}]$ & $\sigma$ & $v[\mathrm{rad} / \mathrm{s}]$ \\
\hline 1 & -5 & $-\pi / 4$ & -5 & $-\pi / 4$ \\
\hline 2 & 0.3 & $-\pi / 6$ & 0.3 & $-\pi / 6$ \\
\hline 3 & 0.3 & $-\pi / 12$ & 0.3 & $-\pi / 12$ \\
\hline 4 & 0.3 & 0 & 0.3 & 0 \\
\hline 5 & 0.3 & $\pi / 12$ & 0.3 & $\pi / 12$ \\
\hline 6 & 0.3 & $\pi / 6$ & 0.3 & $\pi / 6$ \\
\hline 7 & 5 & $\pi / 4$ & 5 & $\pi / 4$ \\
\hline
\end{tabular}

Regarding the fuzzy observer, in this work, we consider only two input variables including the swing angle of the payload $\theta$ and its velocity $\dot{\theta}$. Moreover, in the 
construction of the fuzzy system, we propose to utilize two membership functions, comprising Gaussian function

$$
\mu_{A_{j}^{i}}\left(x_{j}\right)=\frac{1}{1+\exp \left(-\sigma\left(x_{j}-v\right)\right)},
$$

for $i=\{1,7\}$ and sigmoid function

$$
\mu_{A_{j}^{i}}\left(x_{j}\right)=\exp \left(-0.5\left(x_{j}-v\right)^{2} / \sigma^{2}\right)
$$

for $i=\{2, \ldots, 6\}$. And the parameters $(v, \sigma)$ of the membership functions are specified in

Table 1. It is noticed that we have only two input variables $x_{j}=(\theta, \dot{\theta}), j=\{1,2\}$.

For the adaptation mechanism, $\Gamma_{i}(i=1, \ldots, 4)$ was set to 0.01 , and the initial values of the estimated adjustable parameters of the fuzzy observer were all set to $0.1, \hat{\phi}_{i}(0)=0.1$.

\subsection{Simulations}

We now investigate the simulation results including the position of the trolley, the swing angle of the payload and the control force as pictorially illustrated in Figures $3-5$. It was expected that the trolley horizontally travelled $0.6 \mathrm{~m}$ from the initial location. It can be clearly seen in Fig. 3 that given the known and certain system parameters, the HSMC law drove the trolley to reach the destination after about $2.5 \mathrm{~s}$ without overshoot. Comparatively, the proposed AFHSMC scheme, which, though, had to adaptively estimate the system parameters by the use of the fuzzy observer, drove the trolley to reach the destination after around $3 \mathrm{~s}$. Although the proposed approach caused the overshoot in the output, it is apparently trivial. The results shown in Fig. 3 illustrate that both the deterministic HSMC and proposed AFHSMC methods well tracked the desired position of the trolley.

In terms of the swing angle of the payload as demonstrated in Fig. 4, both the HSMC and AFHSMC algorithms, at first $4 \mathrm{~s}$, swung the object, though the maximum 
swing angle is about $0.08 \mathrm{rad}$. More importantly, as expected, due to requirement of time consumed in estimating the system parameters, the swing angle output as a result of the AFHSMC technique is a little bit lag as compared with that of the HSMC method. Nevertheless, ultimately, both approaches eliminated the swing angle of the payload after approximate $4 \mathrm{~s}$, when the trolley reached the destination.

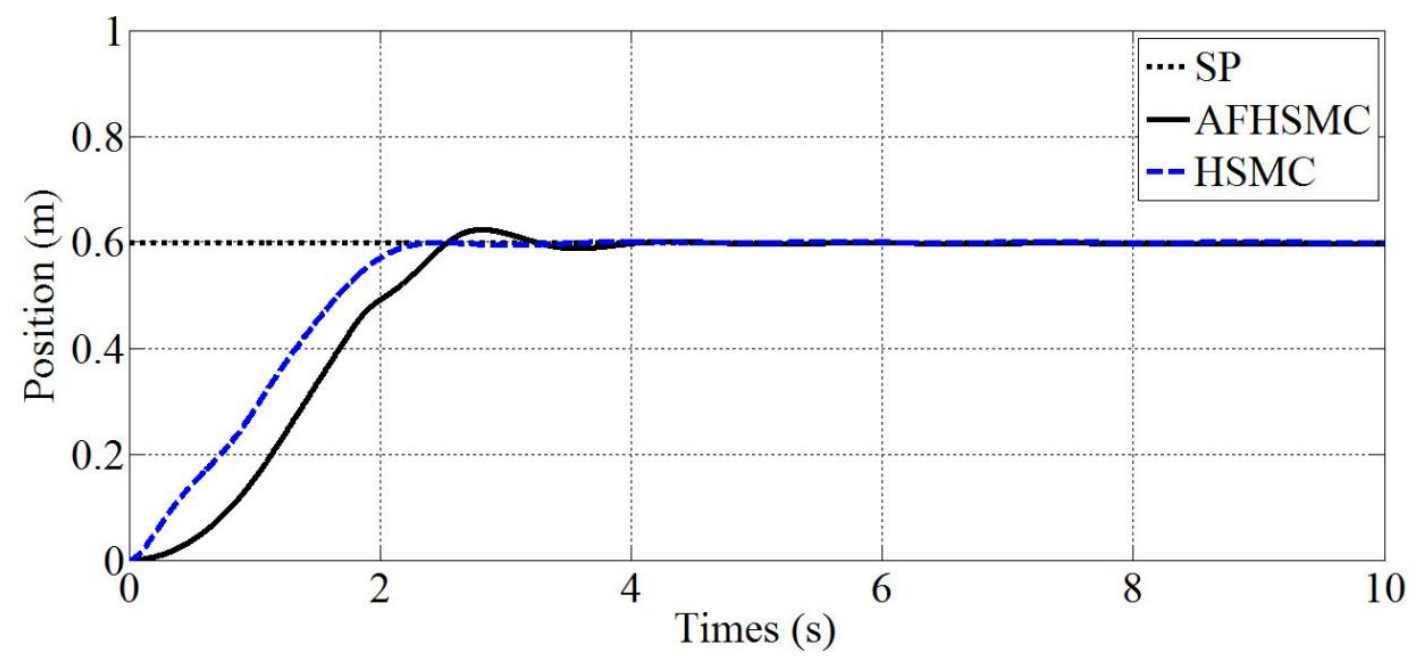

Figure 3. Position of the trolley in the simulation

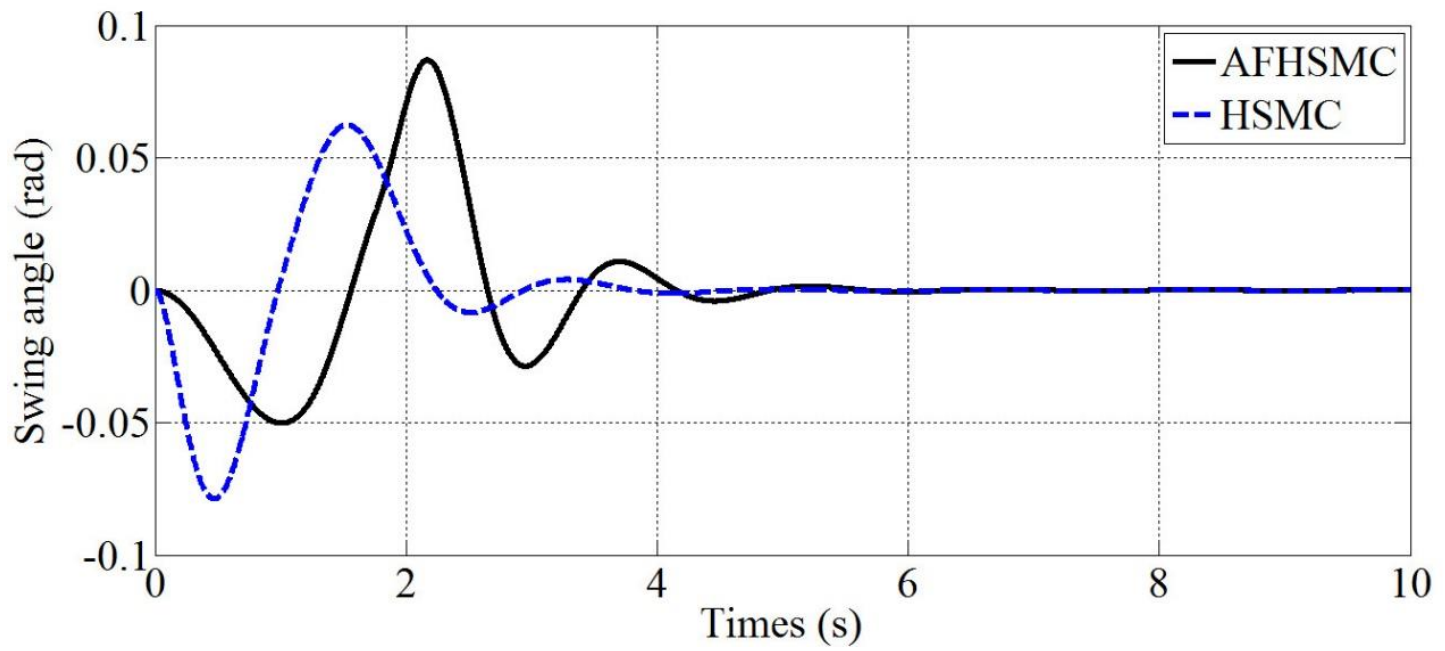

Figure 4. Swing angle of the payload in the simulation 


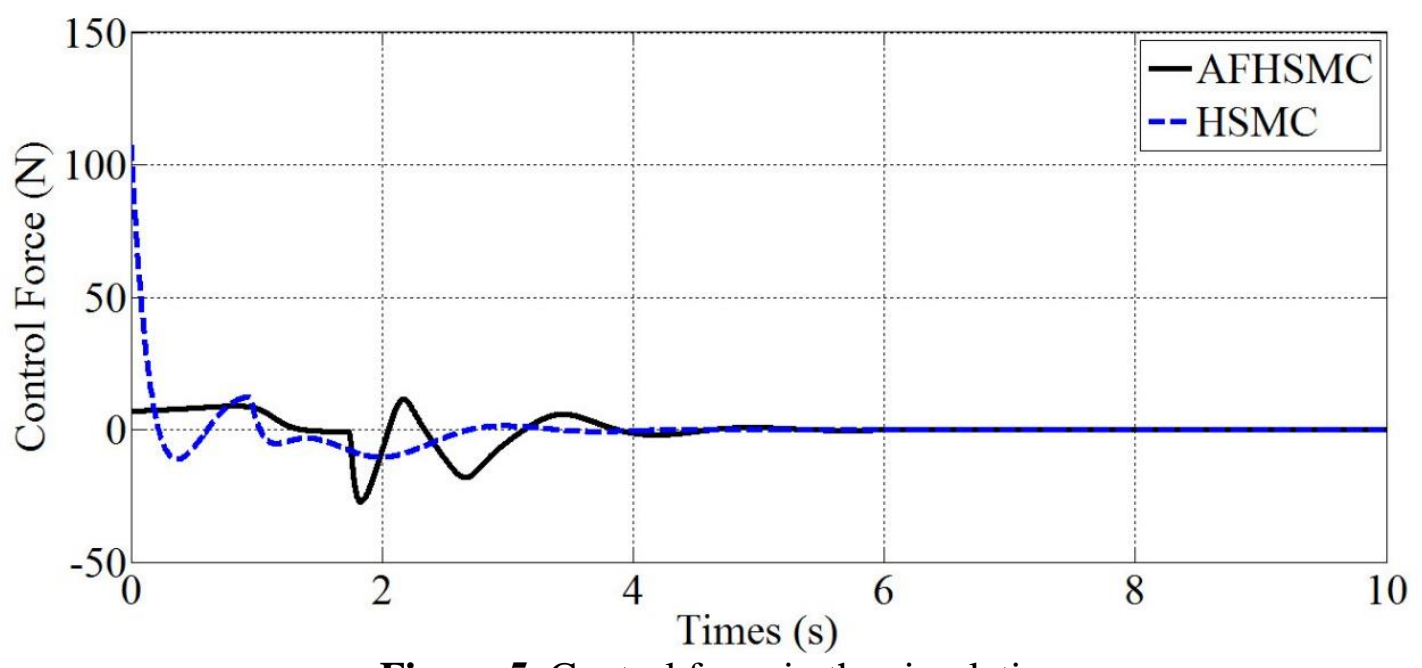

Figure 5. Control force in the simulation

In corresponding to the position of the trolley and the swing angle of the object, the control forces required in both the control laws are highly comparable as can be seen in Fig. 5. It is emphasized that given no prior information of the overhead crane system parameters, the proposed AFHSMC approach is capable of adaptively estimating those parameters, which eventually leads to the actuated and unactuated outputs highly compared with those obtained by the HSMC scheme in ideal scenarios. That is, the AFHSMC law is highly practical as demonstrated in the following section by the laboratory experiments.

\subsection{Real experiments}

To experimentally evaluate effectiveness of the proposed method, we implemented the algorithm in a real-time overhead crane system in a laboratory as illustrated in Fig. 2. In this example system, the trolley was driven by a three-phase asynchronous motor, where the motor was powered by the OMRON inverter 3G3JX. The position of the trolley was measured by the encoder E40S6-1024-3-T-24. Furthermore, the swing angle of the payload and its angular velocity were gathered by the sensor MPU6050. Regarding the 
central control unit, we implemented the proposed approach on the STM32F4 microcontroller.

Under the control of the AFHSMC law, the position of the trolley, the swing angle of the payload and the control force were recorded over time and are now plotted in Figures 6, 7 and 8, respectively. It is noted that the real-life overhead crane system operated under realistic nonlinearity of the actuators, external disturbances and system parameter uncertainty. Nonetheless, the results obtained by the proposed algorithm are appealing. It can be clearly seen that the trolley gradually reached the destination after about $5 \mathrm{~s}$ without overshoot as demonstrated in Fig. 6. In the first second, the controller made the object to swing up to $0.15 \mathrm{rad}$. However, the swing angle of the payload quickly declined to $0.05 \mathrm{rad}$ and almost disappeared after about $5 \mathrm{~s}$ as can be seen in Fig. 7. In contrast to the simulation, the control force in Fig. 8 gradually reduced from beginning and reached to zero at about $5 \mathrm{~s}$ when the trolley stopped.

Though we set the system parameters in the simulation similarly to those in the real-time system, the realistic trolley needed about $5 \mathrm{~s}$ to reach the destination while the synthetic one needed about 3 s. That can be understood by the ability of the microcontroller in the laboratory experiments as compared with that to the desktop.

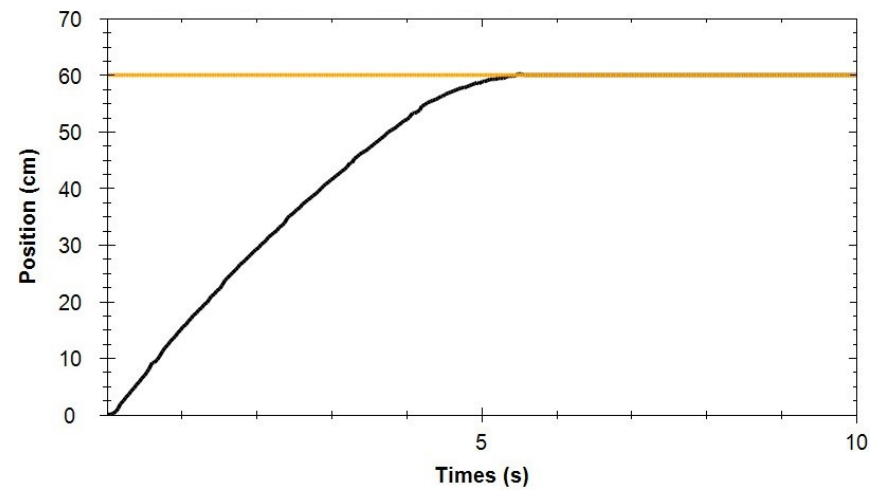

Figure 6. Position of the trolley in the realistic experiment 


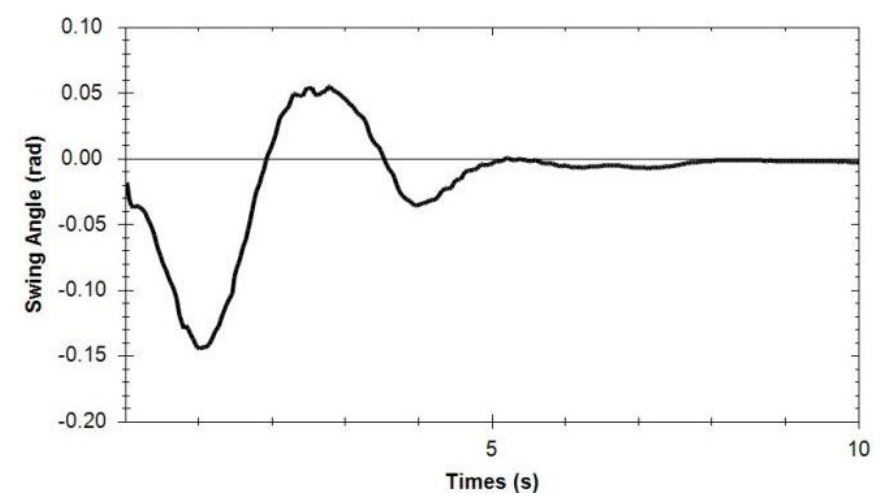

Figure 7. Swing angle of the payload in the realistic experiment

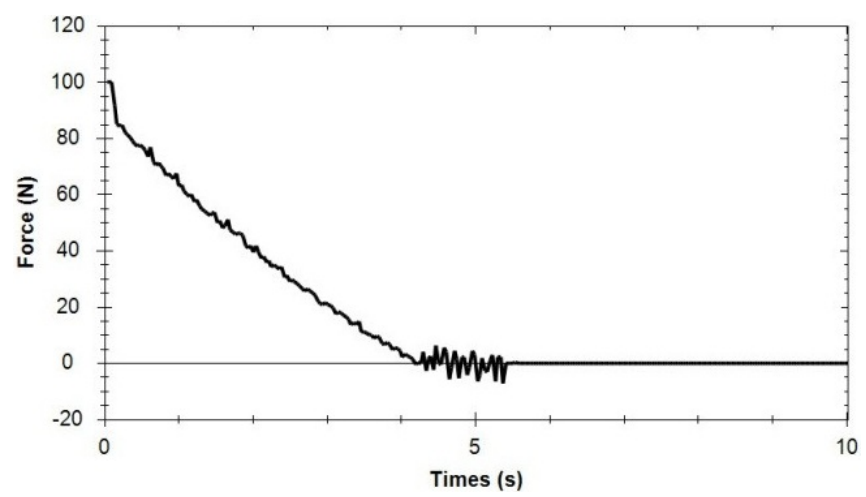

Figure 8. Control force in the realistic experiment

\section{Conclusions}

The paper has proposed to employ the HSMC and fuzzy observer to be deployed in a new but efficient technique to adaptively control an under-actuated overhead crane. The HSMC law enables the system to robustly transport a payload to a destination despite its unknown external disturbances and nonlinearities. More importantly, under influence of the system uncertainties, the parameters of the control law are adaptively estimated, which is derived from the Lyapunov theory. The adaptive mechanism, which theoretically proves the stability of the proposed control scheme, guarantees the crane to be able to effectively work under uncertain conditions. Implementations of the proposed algorithm in both the synthetic simulations and real-life experiments has verified effectiveness of the AFHSMC approach. 


\section{Disclosure statement}

No potential conflict of interest was reported by the authors.

\section{References.}

1. Bartolini, G., Pisano, A. and Usai, E., "Second-order sliding-mode control of container cranes," Automatica, Vol. 38, No. 10, pp. 1783-1790, 2002.

2. Butler, H., Honderd, G. and Amerongen, J. V., "Model reference adaptive control of a gantry crane scale model,” IEEE Control Systems, Vol. 11, No. 1, pp. 57-62, 1991.

3. Jisup, Y., Nation, S., Singhose, W. and Vaughan, J., "Control of crane payloads that bounce during hoisting," IEEE Transactions on Control Systems Technology, Vol. 22, No. 3, pp. 1233-1238, 2014.

4. Lee, H., "Motion planning for three-dimensional overhead cranes with high-speed load hoisting,” International Journal of Control, Vol. 78, No. 12, pp. 37-41, 2007.

5. Omar, H. M. and Nayfeh, A. H., "Anti-swing control of gantry and tower cranes using fuzzy and time delayed feedback with friction compensation," Shock and Vibration, Vol. 12, No. 2, pp. 73-89, 2005.

6. Yu, J., Lewis, F. L., and Huang, T., "Nonlinear Feedback Control of a Gantry Crane," Proceeding of American Control Conference, pp. 4310-4315, 1995.

7. Park, H., Chwa, D., and Hong, K., “A feedback linearization control of container cranes: Varying rope length," International Journal of Control, Automation and Systems, Vol. 5, No. 4, pp. 379-387, 2007.

8. Le, T. A., Kim, G. H., Kim, M. Y., and Lee, S. G., "Partial Feedback Linearization Control of Overhead Cranes with Varying Cable Lengths," International Journal of Precision Engineering and Manufacturing, Vol. 13, No. 4, pp. 501-507, 2012. 
9. Le, T. A., Lee, S., Dang, V., Moon, S. and Kim, B., "Partial feedback linearization control of a three-dimensional overhead crane," International Journal of Control, Automation and Systems, Vol. 11, No. 4, pp. 718-727, 2013.

10. Mahfouf, M., Kee, C. H., Abbod, M. F., and Linkens, D. A., "Fuzzy Logic-Based Anti-Sway Control Design for Overhead Cranes," Neural Computing \& Applications, Vol. 9, No. 1, pp. 38-43, 2000.

11. Wang, L., Zhang, H., and Kong, Z., "Anti-swing Control of Overhead Crane based on Double Fuzzy Controllers," Proceeding of Chinese Control and Decision Conference, pp. 981-986, 2015.

12. Shyu, K. K., Jen, C. L., and Shang, L. J., "Design of Sliding-mode Controller for Anti-swing Control of Overhead Cranes," IECON Proceeding of Annual Conference of IEEE Industrial Electronics Society, pp. 147-152, 2005.

13. Qian, D., Yi, J., and Zhao, D., "Control of Overhead Crane Systems by Combining Sliding Mode with Fuzzy Regulator,” IFAC Proceedings Volumes, Vol. 44, No. 1, pp. 9320-9325, 2011.

14. Le, T. A., Lee, S. G., and Moon, S. C., "Partial Feedback Linearization and Sliding Mode Techniques for 2D Crane Control," Transactions of the Institute of Measurement and Control, Vol. 36, No. 1, pp. 78-87, 2013.

15. Xu, W., Zheng, X., Liu, Y., Zhang, M. and Luo, Y., “Adaptive dynamic sliding mode control for overhead cranes," Proceeding of Chinese Control Conference, pp. $3287-3292,2015$.

16. Wang, W., Yi, J., Zhao, D., and Liu, D., "Design of a Stable Sliding-mode Controller for a Class of Second-order Underactuated Systems," IEE Proceedings Control Theory and Applications, Vol. 151, No. 6, pp. 243-250, 2004. 
17. Qian, D., Yi, J., and Zhao, D., "Hierarchical Sliding Mode Control for a Class of SIMO under-actuated Systems," Control and Cybernetics., Vol. 37, No. 1, pp. 160$175,2008$.

18. Yang, J. H. and Yang, K. S., "Adaptive Coupling Control for Overhead Crane Systems," Mechatronics, Vol. 17, No. 2-3, pp. 143-152, 2007.

19. Yang, J. H. and Shen, S. H., "Novel Approach for Adaptive Tracking Control of a 3-D Overhead Crane System," Journal of Intelligent \& Robotic Systems, Vol. 62, No. 1, pp. 59-80, 2011.

20. Le, T. A., Lee, S. G., Luong, N. C., and Kim, D. H., "Model Reference Adaptive Sliding Mode Control for Three Dimensional Overhead Cranes,” International Journal of Precision Engineering and Manufacturing, Vol. 14, No. 8, pp. 1329-1338, 2013.

21. Kim, E., “A Fuzzy Disturbance Observer and its Application to Control," IEEE Transactions on Fuzzy Systems, Vol. 10, No. 1, pp. 77-84, 2002.

22. Park, M. S., Chwa, D., and Hong, S. K., “Antisway Tracking Control of Overhead Cranes with System Uncertainty and Actuator Nonlinearity using an Adaptive Fuzzy Sliding-mode Control," IEEE Transactions on Industrial Electronics., Vol. 55, No. 11, pp. 3972-3984, 2008.

23. Park, M., Chwa, D., and Eom, M., "Adaptive Sliding-Mode Antisway Control of Uncertain Overhead Cranes With High-Speed Hoisting Motion,” IEEE Transactions on Fuzzy Systems, Vol. 22, No. 5, pp. 1262-1271, 2014.

24. Hung, L. and Chung, H., "Decoupled control using neural network-based slidingmode controller for nonlinear systems," Expert Systems with Applications, Vol. 32, No. 4, pp. 1168-1182, 2007. 
25. Hung, L. and Chung, H., "Decoupled sliding-mode with fuzzy-neural network controller for nonlinear systems," International Journal of Approximate Reasoning, Vol. 46, No. 1, pp. 74-97, 2007.

26. Tsai, C., Wu, H.L. and Chuang, K., "Intelligent sliding-mode motion control using fuzzy wavelet networks for automatic 3D overhead cranes," Proceeding of SICE Annual Conference, pp. 1256-1261, 2012.

27. Le, H. X., Nguyen, T. V., Le, V. A., Vu, N. T. T., Phan, M. X., “Adaptive Backstepping Hierarchical Sliding Mode Control for Uncertain 3D Overhead Crane Systems," Proceeding of IEEE International Conference on System Science and Engineering, pp. 438-443, 2017.

28. Le, V. A., Le, H. X., Vu, D. T., Pham, V. T., Le, A. T., and Hoang, M. C., "Designing an Adaptive Controller for 3D Overhead Cranes using Hierarchical Sliding Mode and Neural Network," Proceeding of IEEE International Conference on System Science and Engineering, (in press), 2018.

29. Hoang, T. T. U., Pham, D. T., Le V. A., and Phan, X. M., “Adaptive Neural Networks Sliding Mode Backstepping Control For 3DOF Surface Ship with Uncertain Model", Proceeding of IEEE International Conference on System Science and Engineering, (in press), 2018.

30. Le, T. A., Hoang, M. C., Pham, V. T., Luong, C. N., Vu, D. T., and Le, V. A., “Adaptive Neural Network Sliding Mode Control of Shipboard Container Cranes Considering Actuator Backlash”, Mechanical Systems and Signal Processing, Vol. 112, pp. 233-250, 2018.

31. Slotine, J. J. and Li, W., “Applied Nonlinear Control,”, Englewood Cliffs, N.J Prentice Hall, 1991.

32. Wang, N., Karimi, H. R., Li, H. and Su, S. F., "Accurate Trajectory Tracking of 
Distributed Surface Vehicles: A Finite-Time Control Approach", IEEE/ASME Transactions on Mechatronics, 2019, Early Access, DOI: https://doi.org/10.1109/TMECH.2019.2906395.

33. Le, V. A., Le, H. X., Nguyen, L. and Phan, M. X., "An Efficient Adaptive Hierarchical Sliding Mode Control Strategy using Neural Network for 3D Overhead Cranes", International Journal of Automation and Computing, 2019, Early Access, DOI: $10.1007 / \mathrm{s} 11633-019-1174-y$. 